\title{
Reduction of speckle in digital holography by discrete Fourier filtering
}

\author{
Jonathan Maycock, Bryan M. Hennelly, and John B. McDonald \\ Department of Computer Science, National University of Ireland, Maynooth, County Kildare, Ireland \\ Yann Frauel \\ Departamento de Ciencias de la Computación, Instituto de Investigaciones en Matemáticas Aplicadas y Sistemas, \\ Univeŕsidad Nacional Autónoma de México, Apartado Postal 20-726 Admon. No. 20, Del. Alvaro Obregón \\ 01000 México Distrito Federal, México \\ Albertina Castro \\ Instituto Nacional de Astrofísica, Óptica y Electrónica, Apartado Postal 51, Puebla, Puebla 72000, Mexico \\ Bahram Javidi \\ Department of Electrical and Computer Engineering, University of Connecticut, U-157, Storrs,
} Connecticut 06269, USA

\section{Thomas J. Naughton}

Department of Computer Science, National University of Ireland, Maynooth, County Kildare, Ireland, and University of Oulu, RF Media Laboratory, Oulu Southern Institute, Vierimaantie 5, 84100 Ylivieska, Finland

\author{
Received September 21, 2006; accepted December 8, 2006; \\ posted January 2, 2007 (Doc. ID 75293); published May 9, 2007
}

\begin{abstract}
We present a digital signal processing technique that reduces the speckle content in reconstructed digital holograms. The method is based on sequential sampling of the discrete Fourier transform of the reconstructed image field. Speckle reduction is achieved at the expense of a reduced intensity and resolution, but this tradeoff is shown to be greatly superior to that imposed by the traditional mean and median filtering techniques. In particular, we show that the speckle can be reduced by half with no loss of resolution (according to standard definitions of both metrics). (C) 2007 Optical Society of America

OCIS codes: $100.2000,030.4280,030.6140,090.2880,070.2580$.
\end{abstract}

\section{INTRODUCTION}

Speckle occurs when coherent light is diffused by an optically rough surface. ${ }^{1-5}$ It degrades the quality of the rendered images and makes the accurate viewing of small details very difficult. The speckle problem is always present in holography since it uses coherent light. There have been some recent contributions to the reduction of speckle in digital hologram (DH) reconstructions. ${ }^{6,7}$ These methods use diffusers to partially reduce the coherence of the illumination and thus achieve a reduction in speckle. Another approach put forward for the removal of speckle was based on the maximum-likelihood technique and used a general model for image reflectivity. ${ }^{8}$

Dainty and Welford ${ }^{9}$ optically reduced speckle in image plane hologram reconstructions by rotating a circular aperture in the Fourier plane of the image. Hariharan and Hegedus ${ }^{10}$ extended the method by superimposing the exposures from bandpassed filtered images of a diffuse object. By interpreting our $\mathrm{DH}$ as the complex wave field at a particular intermediate plane in the coherent imaging speckle removal system, we can apply the discrete analog of this process to DHs. Furthermore, this digital signal processing (DSP) technique, which we call discrete Fourier filtering (DFF), offers a number of considerable advantages both to its optical counterpart and to other existing DSP methods. These advantages are discussed after the analysis is presented.

The optical setup [in Fig. 1(a)], upon which our technique is modeled, is that used in Refs. 9 and 10 except that we include the position of our hologram plane. The wavefront emanating from the diffuse object propagates through the $4 f$ system in which an aperture is placed in the Fourier plane. The aperture is moved between each of several exposures, and the intensities in the image plane are integrated over the exposures. This leads to a specklereduced image plane signal. We capture our inline Fresnel DHs ${ }^{11-13}$ using phase-shift interferometry. ${ }^{14}$ The effect of capturing the DH introduces an extra aperture between the object and the first lens, namely, the recording CCD sensor. We assume for simplicity that the effect 


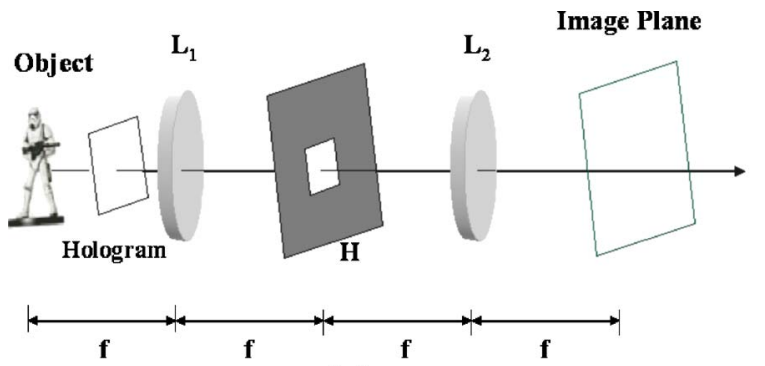

(a)

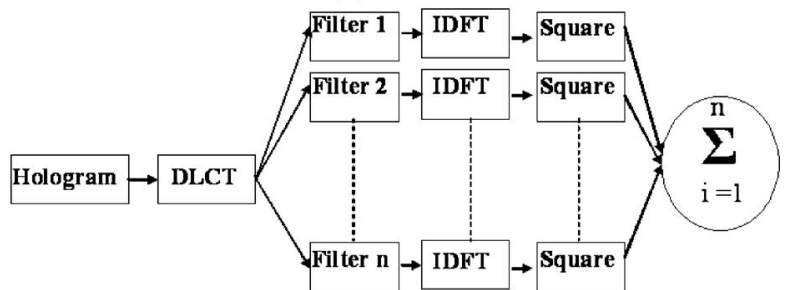

(b)

Fig. 1. (Color online) (a) Coherent optical imaging system with a moving filter in the Fourier plane. The hologram plane denotes the location that our DH would occupy in such a system. (b) Schematic of the DFF technique, which is the discrete analog of the optical technique in (a), starting from the hologram plane. DLCT, discrete linear canonical transform; IDFT, inverse discrete Fourier transform.

of this is only to band limit the object wave field. We then digitally implement the rest of the setup in the paraxial regime to a high degree of accuracy.

\section{ANALYSIS}

The following analysis is based on previous work done by Lowenthal and Arsenault ${ }^{15}$ and Hariharan and Hegedus, ${ }^{10}$ and for convenience follows their notation. A plane, $f(\mathbf{r})$, immediately in front of a diffuse nonuniform object, which is illuminated by a coherent beam can be expressed as the product of two terms,

$$
f(\dot{\mathbf{r}})=t(\mathbf{r}) d(\mathbf{r})
$$

where $d(\mathbf{r})$ is a uniform diffuser, $t(\mathbf{r})$ is a transparency that modulates the diffuser, and $\mathbf{r}$ is a vector $(x, y)$ in the plane in front of the object. The image plane amplitude $g(\mathbf{r})$ is defined as $g(\mathbf{r})=f(\mathbf{r}) * h(\mathbf{r})$, where $h(\mathbf{r})$ is the amplitude impulse response of the imaging system and $*$ denotes a convolution. It will be shown that the average power spectrum of the image intensity is related to the autocorrelation of the image intensity. ${ }^{16}$ We will also show that the power spectrum of the image intensity can be spilt into two terms. The first being the power spectrum of the image itself, and the second being the power spectrum of the speckle, which we want to reduce. The autocorrelation of $f(\mathbf{r})$ can be written ${ }^{15}$

$$
R_{f f}\left(\mathbf{r}_{1}, \mathbf{r}_{2}\right)=\left\langle f\left(\mathbf{r}_{1}\right) f^{*}\left(\mathbf{r}_{2}\right)\right\rangle=\left\langle t\left(\mathbf{r}_{1}\right) d\left(\mathbf{r}_{1}\right) t^{*}\left(\mathbf{r}_{2}\right) d^{*}\left(\mathbf{r}_{2}\right)\right\rangle,
$$

where $\left\langle f\left(\mathbf{r}_{1}\right) f^{*}\left(\mathbf{r}_{2}\right)\right\rangle$ is an expectation, which is defined as $\langle X\rangle=\int_{-\infty}^{\infty} x P_{x}(x) \mathrm{d} x$, where $P_{x}(x)$ is the probability density function for $X$ having a value of $x$. Since $t$ is not a random function, it can be extracted from the expectation, as in

$$
R_{f f}\left(\mathbf{r}_{1}, \mathbf{r}_{2}\right)=t\left(\mathbf{r}_{1}\right) t^{*}\left(\mathbf{r}_{2}\right)\left\langle d\left(\mathbf{r}_{1}\right) d^{*}\left(\mathbf{r}_{2}\right)\right\rangle=t\left(\mathbf{r}_{1}\right) t^{*}\left(\mathbf{r}_{2}\right) R_{d d}\left(\mathbf{r}_{1}, \mathbf{r}_{2}\right) .
$$

$d(\mathbf{r})$ is considered to be stationary and Gaussian with zero mean and its autocorrelation may be approximated by a delta function,

$$
R_{d d}\left(\mathbf{r}_{1}, \mathbf{r}_{2}\right)=\left\langle d\left(\mathbf{r}_{1}\right) d^{*}\left(\mathbf{r}_{2}\right)\right\rangle=\delta\left(\mathbf{r}_{1}-\mathbf{r}_{2}\right) .
$$

If $j$ exposures are made with a shift of the aperture between them, the resultant intensity $I(\mathbf{r})$ at any point in the image is the sum of the intensities at this point due to the individual $j$ exposures,

$$
I(\mathbf{r})=\sum_{j} I_{j}(\mathbf{r})
$$

where $I_{j}(\mathbf{r})=\left|g_{j}(\mathbf{r})\right|^{2}$, where $g_{j}(\mathbf{r})=f(\mathbf{r}) * h_{j}(\mathbf{r})$, and where $h_{j}(\mathbf{r})$ the impulse response of the system for the $j$ th position of the aperture in the Fourier plane. Due to the linearity of convolution, the autocorrelation $R_{I I}\left(\mathbf{r}_{1}, \mathbf{r}_{2}\right)$ of the image intensity can be written as

$$
R_{I I}\left(\mathbf{r}_{1}, \mathbf{r}_{2}\right)=\sum_{m, n} R_{I_{m} I_{n}}\left(\mathbf{r}_{1}, \mathbf{r}_{2}\right),=\sum_{m, n}\left\langle\left|g_{m}\left(\mathbf{r}_{1}\right)\right|^{2}\left|g_{n}\left(\mathbf{r}_{2}\right)\right|^{2}\right\rangle,
$$

where the summation is taken over all values of $m$ and $n$, and each term is the result of an expectation.

For two complex, Gaussian variables $X_{1}$ and $X_{2}$, with zero mean, the expectation $\left\langle\left|X_{1}\right|^{2}\left|X_{2}\right|^{2}\right\rangle$ is given by a theorem of Reed $^{17}$ to be

$$
\left\langle\left|X_{1}\right|^{2}\left|X_{2}\right|^{2}\right\rangle=\left\langle\left|X_{1}\right|^{2}\right\rangle\left\langle\left|X_{2}\right|^{2}\right\rangle+\left|\left\langle X_{1} X_{2}^{*}\right\rangle\right|^{2} .
$$

As we are dealing with expectations, the autocorrelation of the image intensity can be rewritten as

$$
\begin{aligned}
R_{I I}\left(\mathbf{r}_{1}, \mathbf{r}_{2}\right) & =\sum_{m, n}\left\langle\left|g_{m}\left(\mathbf{r}_{1}\right)\right|^{2}\right\rangle\left\langle\left|g_{n}\left(\mathbf{r}_{2}\right)\right|^{2}\right\rangle+\sum_{m, n}\left|\left\langle g_{m}^{*}\left(\mathbf{r}_{1}\right) g_{n}\left(\mathbf{r}_{2}\right)\right\rangle\right|^{2}, \\
& =\sum_{m, n}\left\langle I_{m}\left(\mathbf{r}_{1}\right)\right\rangle\left\langle I_{n}\left(\mathbf{r}_{2}\right)\right\rangle+\sum_{m, n}\left|R_{g_{m} g_{n}}\left(\mathbf{r}_{1}, \mathbf{r}_{2}\right)\right|^{2}
\end{aligned}
$$

The average power spectrum of the image intensity is $\Omega(\mathbf{u}, \mathbf{u})$, where $\Omega\left(\mathbf{u}_{1}, \mathbf{u}_{2}\right)$ is the double Fourier transform (FT) of the autocorrelation of the image intensity, ${ }^{15}$

$$
\begin{aligned}
\Omega\left(\mathbf{u}_{1}, \mathbf{u}_{2}\right) & =F\left\{R_{I I}\left(\mathbf{r}_{1}, \mathbf{r}_{2}\right)\right\}, \\
& =F\left\{\sum_{m, n}\left\langle I_{m}\left(\mathbf{r}_{1}\right)\right\rangle\left\langle I_{n}\left(\mathbf{r}_{2}\right)\right\rangle\right\}+F\left\{\sum_{m, n}\left|R_{g_{m} g_{n}}\left(\mathbf{r}_{1}, \mathbf{r}_{2}\right)\right|^{2}\right\},
\end{aligned}
$$

where $F$ is the double FT operator, defined by Lowenthal and Arsenault ${ }^{15}$ as $F\left\{R_{I I}\left(\mathbf{r}_{1}, \mathbf{r}_{2}\right)\right\}$ $=\int_{-\infty}^{\infty} \int_{-\infty}^{\infty} R_{I I}\left(\mathbf{r}_{1}, \mathbf{r}_{2}\right) e^{-i 2 \pi\left(\mathbf{u}_{1} \cdot \mathbf{r}_{1}-\mathbf{u}_{2} \cdot \mathbf{r}\right)} \mathrm{d} \mathbf{r}_{1} \mathrm{~d} \mathbf{r}_{2}$. In the second part of Eq. (9), the first term, which we denote $\Omega_{1}\left(\mathbf{u}_{1}, \mathbf{u}_{2}\right)$ represents the power spectrum of the image, and the second term, $\Omega_{2}\left(\mathbf{u}_{1}, \mathbf{u}_{2}\right)$, represents the power spectrum of the speckle.

It has also been shown by Lowenthal and Arsenault ${ }^{15}$ that the average intensity $\langle I(\mathbf{r})\rangle$ in the image of a coherently illuminated diffuse object is given by the relation $\langle I(\mathbf{r})\rangle=|t(\mathbf{r})|^{2} *|h(\mathbf{r})|^{2}$. This result means that $\Omega_{1}\left(\mathbf{u}_{1}, \mathbf{u}_{2}\right)$ can be given by 


$$
\begin{aligned}
\Omega_{1}\left(\mathbf{u}_{1}, \mathbf{u}_{2}\right)= & \sum_{m, n}\left[T\left(\mathbf{u}_{1}\right) * T^{*}\left(-\mathbf{u}_{1}\right)\right]\left[H_{m}\left(\mathbf{u}_{1}\right) * H_{m}^{*}\left(-\mathbf{u}_{1}\right)\right] *[T \\
& \left.\times\left(-\mathbf{u}_{2}\right) * T^{*}\left(\mathbf{u}_{2}\right)\right]\left[H_{n}\left(-\mathbf{u}_{2}\right) * H_{n}^{*}\left(\mathbf{u}_{2}\right)\right],
\end{aligned}
$$

where $T(\mathbf{u})$ is the FT of $t(\mathbf{r})$, and $H(\mathbf{u})$ is the FT of $h(\mathbf{r})$. We now evaluate the other term $\Omega_{2}\left(\mathbf{u}_{1}, \mathbf{u}_{2}\right)$ that gives the power spectrum of the speckle. ${ }^{10}$ For two linear systems with input $f(\mathbf{r})$, impulse responses $h_{m}(\mathbf{r}), h_{n}(\mathbf{r})$ and outputs $g_{m}(\mathbf{r}), g_{n}(\mathbf{r})$, the cross correlation of the outputs can be written in terms of the cross-correlation of the inputs. Using this result and the right-hand side of Eq. (9), we have ${ }^{15}$

$$
\Omega_{2}\left(\mathbf{u}_{1}, \mathbf{u}_{2}\right)=\sum_{m, n} F\left\{\left|R_{f f}\left(\mathbf{r}_{1}, \mathbf{r}_{2}\right) *_{\mathbf{r}_{1}} h_{m}\left(\mathbf{r}_{1}\right) *_{\mathbf{r}_{2}} h_{n}^{*}\left(\mathbf{r}_{2}\right)\right|^{2}\right\}
$$

where the subscripts $\mathbf{r}_{1}$ and $\mathbf{r}_{2}$ mean that the convolution operation is carried out on the variables $\mathbf{r}_{1}$ and $\mathbf{r}_{2}$, respectively, the other variable being treated as a constant, and $R_{f f}\left(\mathbf{r}_{1}, \mathbf{r}_{2}\right)$ is the autocorrelation function of the object amplitude $f(\mathbf{r})$.

Now, $\Omega_{2}\left(\mathbf{u}_{1}, \mathbf{u}_{2}\right)$, which is the power spectrum of the speckle, can be written as

$$
\begin{aligned}
\Omega_{2}\left(\mathbf{u}_{1}, \mathbf{u}_{2}\right)= & \sum_{m, n}\left[\Gamma\left(\mathbf{u}_{1}, \mathbf{u}_{2}\right) H_{m}\left(\mathbf{u}_{1}\right) H_{n}^{*}\left(-\mathbf{u}_{2}\right)\right] *\left[\Gamma^{*}\left(-\mathbf{u}_{1},-\mathbf{u}_{2}\right)\right. \\
& \left.\times H_{m}^{*}\left(-\mathbf{u}_{1}\right) H_{n}\left(\mathbf{u}_{2}\right)\right]
\end{aligned}
$$

where $\Gamma\left(\mathbf{u}_{1}, \mathbf{u}_{2}\right)$ is the double FT of $R_{f f}\left(\mathbf{r}_{1}, \mathbf{r}_{2}\right)$.

Finally, if Eq. (12) is rewritten with $\mathbf{u}$ replacing $\mathbf{u}_{1}$ and $\mathbf{u}_{2}$ to obtain the average power spectrum of the speckle, $\Gamma\left(\mathbf{u}_{1}, \mathbf{u}_{2}\right)$ becomes the average power spectrum of the object amplitude, which has been shown to be a constant and is equal to the total power in the signal. ${ }^{15}$ The power spectrum of the speckle is rewritten as

$$
\Omega_{2}(\mathbf{u}, \mathbf{u})=\left|R_{t t}(0)\right|^{2} \sum_{m, n}\left[H_{m}(\mathbf{u}) H_{n}^{*}(\mathbf{u})\right] *\left[H_{m}^{*}(-\mathbf{u}) H_{n}(-\mathbf{u})\right] .
$$

From this equation, Lowenthal and Arsenault ${ }^{15}$ have shown that the manner in which the speckle spatial frequencies are distributed does not depend on the signal $t(\mathbf{r})$, but only on the coherent transfer function of the system. Only the total signal power $\left|R_{t t}(0)\right|^{2}$ effects the power spectrum of the speckle. Using this result and modeling our system's aperture as a $2 \mathrm{D}$ rect function. Eq. (13) becomes

$$
\begin{aligned}
\Omega_{2}(\mathbf{u}, \mathbf{u})= & \left|R_{t t}(0)\right|^{2} \sum_{m_{x}, m_{y}, n_{x}, n_{y}} \\
& \times\left[\operatorname{rect}\left(\frac{u_{x}-m_{x} \Delta u_{x}}{w_{x}}, \frac{u_{y}-m_{y} \Delta u_{y}}{w_{y}}\right)\right. \\
& \left.\times \operatorname{rect}\left(\frac{u_{x}-n_{x} \Delta u_{x}}{w_{x}}, \frac{u_{y}-n_{y} \Delta u_{y}}{w_{y}}\right)\right] *[\text { rect } \\
& \times\left(\frac{u_{x}-m_{x} \Delta u_{x}}{-w_{x}}, \frac{u_{y}-m_{y} \Delta u_{y}}{-w_{y}}\right)
\end{aligned}
$$

$$
\left.\times \operatorname{rect}\left(\frac{u_{x}-n_{x} \Delta u_{x}}{-w_{x}}, \frac{u_{y}-n_{y} \Delta u_{y}}{-w_{y}}\right)\right],
$$

where $\mathbf{u}=u_{x} \hat{x}+u_{y} \hat{y}$, and $\Delta u_{x}, \Delta u_{y}$ is the displacement of the aperture between two exposures in the $x$ and $y$ directions, respectively. $w_{x}$ is the width of the rect function in the $x$ direction and $w_{y}$ is the width of the rect function in the $y$ direction. This reduces to

$$
\begin{aligned}
\Omega_{2}(\mathbf{u}, \mathbf{u})= & \left|R_{u}(0)\right|^{2} \sum_{m_{x}, m_{y}, n_{x}, n_{y}}\left[1-\frac{\left|u_{x}-\Delta u_{x}(m+n)\right|}{w_{x}-\Delta u_{x}|m-n|}\right] \\
& \times\left[1-\frac{\left|u_{y}-\Delta u_{y}(m+n)\right|}{w_{y}-\Delta u_{y}|m-n|}\right] .
\end{aligned}
$$

If $w_{x}-\Delta u_{x}|m-n| \leq 0$ or $w_{y}-\Delta u_{y}|m-n| \leq 0$ then the entire expression goes to 0 . In the limiting case of $\Delta u_{x}=0$ and $\Delta u_{y}=0$, the power spectrum of the speckle is at its maximum, being the same as the power spectrum of the speckle given by a single aperture. When $\Delta u_{x} \geq w_{x}$ and $\Delta u_{y} \geq w_{y}$, so that the two positions of the aperture do not overlap, the power spectrum of the speckle is reduced to half the value of the limiting case. ${ }^{10}$ However, the power spectrum of the image does not follow this trend. It follows that for $n$ exposures, the power spectrum of the speckle is $1 / n$ times that obtained using a single exposure. For maximum efficiency, we have set $\Delta u_{x}=w_{x}$ and $\Delta u_{y}=w_{y}$. Since we are limited by the finite extent of the FT of our DH, we are limited in the number of independent exposures. It should be noted that the smaller the exposure, the greater the loss in resolution in the reconstructed image. However, using basic Fourier theory, one may deduce that the bandwidth of the reconstruction intensity is twice the extent of the rect function. For a more thorough analysis of the optical system, please refer to Lowenthal and Arsenault ${ }^{15}$ and Hariharan and Hegedus. ${ }^{10}$

Figure 1(b) shows the DFF technique for reducing speckle. The first step is to numerically compute the propagation from the discrete hologram plane to the discrete Fourier planed. This can be achieved efficiently in one step using a single discrete linear canonical transform. A number of methods to efficiently implement this transform are outlined by Hennelly and Sheridan. ${ }^{18}$ The Fourier plane data are filtered and then inverse discrete Fourier transformed to the image plane where its intensity is stored. This is repeated $n$ times and the resulting $n$ intensities are summed.

\section{METRICS}

Speckle index ${ }^{19,20}$ was chosen as the metric to evaluate the reduction in speckle achievable using the DFF technique. It is calculated as the ratio of standard deviation to the mean in a homogeneous area. In this experiment, the homogeneous area chosen was a section on the chest of a stormtrooper object (see Fig. 3 below). The original reconstruction has a speckle index of 1.02 , and by minimizing this we reduce the speckle content. This speckle reduction is offset by a loss in resolution, which was calculated using a resolution chart. The chart [see Fig. 2(a)] used is modeled on the U.S. Air Force (USAF) 1951 three-bar re- 
solving power test chart. It is $2048 \times 2048$ pixels in size (matching the resolution of the $\mathrm{DH}$ ). The smallest details on the chart [see Fig. 2(b)] start at just a single pixel in width. We define the resolution level as

$$
R=1 / X
$$

where $X$ is the width of the smallest bars on the resolution chart that can be resolved. A filtering technique for given parameters (i.e., aperture size for the DFF technique and neighborhood size for median and mean filtering) is said to cause no loss in resolution if the smallest three bars (both vertical and horizontal) can be resolved. The technique is thus classified as being at resolution level $\frac{1}{1}$. It follows that if the smallest three bars cannot be resolved, but the next smallest can, then a technique for given parameters is classified as being at level $\frac{1}{2}$. Figure 2 (c) shows a zoomed in section $(115 \times 115$ pixels in size $)$ of the resolution chart after application of the DFF technique with an aperture size of $512 \times 512$, and Fig. 2(d) shows the same zoomed in section of the resolution chart

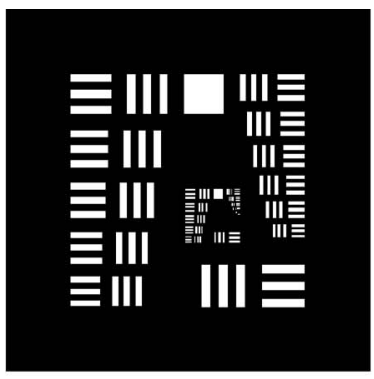

(a)

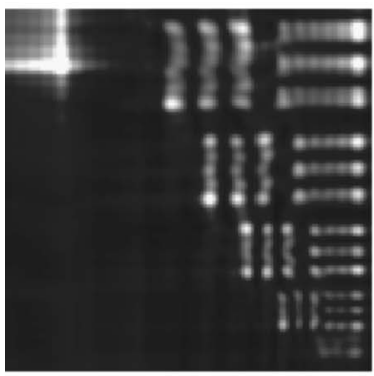

(c)

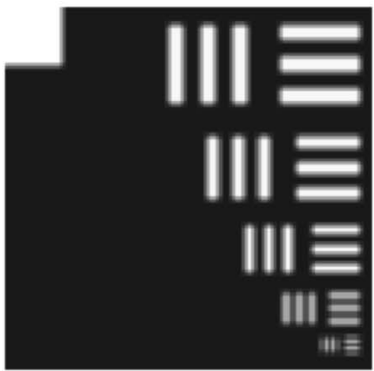

(e)

Fig. 2. Nonfiltered version of the USAF 1951, three-bar resolving power test chart $(2048 \times 2048$ pixels in size $)$, (b) zoomed in $115 \times 115$ pixel region of the chart showing the smallest details on the chart, (c) zoomed in results of applying on (a) the DFF technique with aperture width of 512, (d) zoomed in result of applying on (a) a median filter with a neighborhood size of $3 \times 3$, and (e) zoomed in result of applying on (a) a mean filter with a neighborhood size of $3 \times 3$.

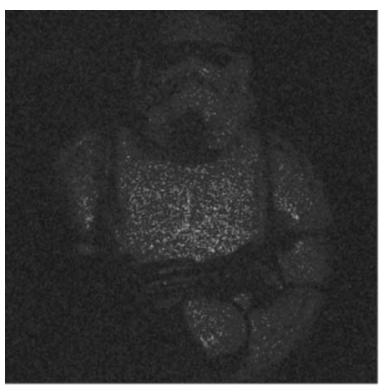

(a)

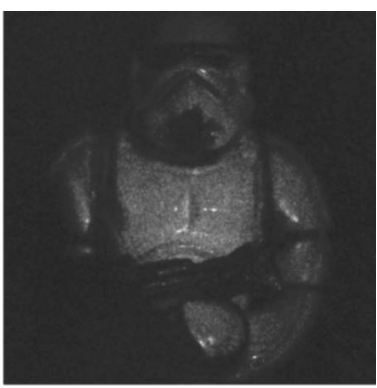

(b)
Fig. 3. (a) Shows the original reconstruction and (b) shows the result of applying the DFF technique.

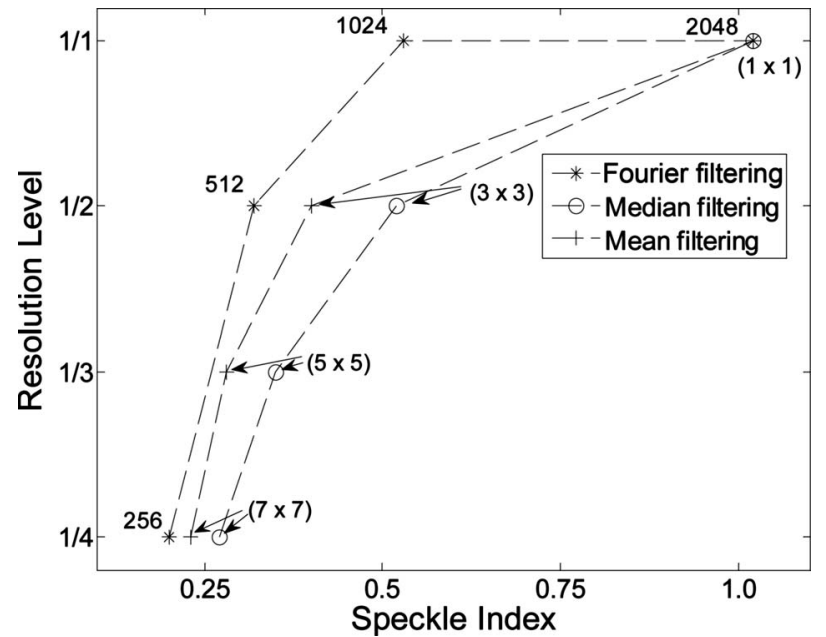

Fig. 4. Graph showing the results of DFF technique (points are labeled with the aperture size used), and the median and the mean filters (points are labeled with the neighborhood sizes used).

after application of the median filtering technique with a neighborhood size of $3 \times 3$. For the parameters given, both of these techniques are said to result in a resolution level of $\frac{1}{2}$.

\section{RESULTS AND DISCUSSION}

Figure 3 shows the results of the DFF technique applied to a DH of a stormtrooper object. In Fig. 3(a), the original reconstruction is shown. It is $2048 \times 2048$ pixels in size, and has a speckle index of 1.02. The application of the discrete Fourier filter to a reconstruction of this $\mathrm{DH}$ is shown in Fig. 3(b). The aperture, $h(r)$, has a width of 256 pixels in size. This results in a speckle index of 0.2 and a resolution level of $\frac{1}{4}$. It is clear that the technique has succeeded in reducing the speckle content.

A graph of the results of applying the DFF technique, median filtering, and mean filtering to a reconstruction of a $\mathrm{DH}$ of the stormtrooper figure is shown in Fig. 4. It shows that the speckle index can be reduced by nearly half with no loss in resolution using the DFF technique. In comparison, using either the median or mean filters to achieve a similar reduction in speckle index, will result in a drop to a resolution level of $\frac{1}{2}$. At each resolution level, the discrete Fourier filter consistently has a lower speckle index than either the median or the mean filters. 
Simple convolution-based noise reduction techniques, more appropriate for additive noise sources, are routinely applied to reconstructions of digital holograms of $3 \mathrm{D}$ objects due to their simplicity, and due to the lack of an appropriate alternative. When the reconstructions are to be used as the basis for scientific, industrial, or medical decisions (as opposed to simply being visually appealing) it is often not appropriate to employ a complicated noise reduction technique whose manipulation of the data cannot be analyzed easily. Where noise reduction techniques have been reported in the literature, those routinely applied to the reconstructions of digital holograms of 3D objects are mean filtering, ${ }^{21-27}$ Gaussian filtering, ${ }^{28}$ median filtering, ${ }^{11,26,27,29,30}$ subsampling, ${ }^{29,31}$ and superposition of different reconstructions. ${ }^{6,23}$

The DFF technique offers a number of significant advantages over its optical counterpart. First, no additional optical preprocessing or postprocessing is necessary in the capture of DHs. Second, the technique has the advantage of being able to efficiently compute discrete Fourier transformations without the need for a bulk optical system. The well-known fast FT may be used to implement our technique within the order of $N \log _{2} N$ steps where $N$ is the number of pixels in the DH. Third, the technique allows for the use of arbitrary filters that would be difficult to fabricate or represent on a spatial light modulator. In future work, we will look at creating more specialized filters (including complex valued ones) in an effort to improve on the results given here. The DFF technique also has an advantage over some existing DSP techniques, ${ }^{6,7,23}$ in that it only requires a single DH. This implies that the method can be applied to all existing $\mathrm{DHs}$ captured previously.

\section{CONCLUSION}

A DSP technique that reduces the speckle content in reconstructed DHs has been presented. It was shown that the speckle index can be reduced by half with no loss of resolution, and further reductions in speckle can be achieved with some loss in resolution. Furthermore, the DFF technique was shown to be superior to the mean and median filters in terms of speckle reduction and loss of resolution. It is important to note that almost any level of speckle reduction can be achieved, but this always needs to be offset against the resulting loss in resolution. We also showed that the technique offers a number of significant advantages over the optical imaging technique upon which it was modeled, and some existing DSP techniques.

\section{ACKNOWLEDGMENTS}

This publication has emanated from research conducted with the financial support of Science Foundation Ireland, Irish Research Council for Science, Engineering and Technology, and Enterprise Ireland.

Corresponding author John B. McDonald's e-mail address is johnmcd@cs.nuim.ie.

\section{REFERENCES}

1. J. C. Dainty, Laser Speckle and Related Phenomena, 2nd ed. (Springer-Verlag, 1984).

2. P. K. Rastogi, Digital Speckle Pattern Interferometry and Related Techniques (Wiley, 2001).

3. T. Kreis, Handbook of Holographic Interferometry: Optical and Digital Methods (Wiley, 2005).

4. J. W. Goodman, Speckle Phenomena: Theory and Applications (Roberts \& Company, 2006).

5. R. F. Patten, B. M. Hennelly, D. P. Kelly, F. T. O'Neill, Y. Liu, and J. T. Sheridan, "Speckle photography: Mixed domain fractional Fourier motion detection," Opt. Lett. 31, 32-34 (2006).

6. J. Garcia-Sucerquia, J. H. Ramírez, and R. Castaneda, "Incoherent recovering of the spatial resolution in digital holography," Opt. Commun. 260, 62-67 (2006).

7. D. Kim, "Reduction of coherent artifacts in dynamic holographic three-dimensional displays by diffractionspecific pseudorandom diffusion," Opt. Lett. 29, 611-613 (2004).

8. N. Bertaux, Y. Frauel, P. Réfrégier, and B. Javidi, "Speckle removal using a maximum-likelihood technique with isoline gray-level regularization,” J. Opt. Soc. Am. A 21, 2283-2291 (2004).

9. J. C. Dainty and W. T. Welford, "Reduction of speckle in image plane hologram reconstruction by use of a moving pupil,” Opt. Commun. 3, 289-294 (1971).

10. P. Hariharan and Z. S. Hegedus, "Reduction of speckle in coherent imaging by spatial frequency sampling," Opt. Acta 21, 345-356 (1974).

11. T. J. Naughton, Y. Frauel, B. Javidi, and E. Tajahuerce, "Compression of digital holograms for three-dimensional object reconstruction and recognition," Appl. Opt. 41, 4124-4132 (2002)

12. J. Maycock, C. M. Elhinney, B. M. Hennelly, T. J. Naughton, J. McDonald, and B. Javidi, "Three-dimensional scene reconstruction of partially occluded objects using digital holograms," Appl. Opt. 45, 2975-2985 (2006).

13. A. Shortt, T. J. Naughton, and B. Javidi, "Compression of digital holograms of three-dimensional objects using wavelets," Opt. Express 14, 2625-2630 (2006).

14. I. Yamaguchi and T. Zhang, "Phase-shifting digital holography," Opt. Lett. 22, 1268-1270 (1997).

15. S. Lowenthal and H. Arsenault, "Image formation for coherent diffuse objects: Statistical properties," J. Opt. Soc. Am. 60, 1478-1483 (1970).

16. A. Papoulis, Probability, Random Variables, and Stochastic Processes (McGraw Hill, 1965).

17. I. S. Reed, "On a moment theorem for complex Gaussian processes," IRE Trans. Inf. Theory 8, 194-195 (1962).

18. B. M. Hennelly and J. T. Sheridan, "Generalizing, optimizing, and inventing numerical algorithms for the fractional Fourier, Fresnel, and linear canonical transforms," J. Opt. Soc. Am. A 22, 917-927 (2005).

19. L. S. Lim, "Techniques for speckle noise removal," Opt. Eng. (Bellingham) 20, 670-678 (1981).

20. T. J. Crimmins, "Geometric filter for speckle reduction," Appl. Opt. 24, 1438-1443 (1985).

21. O. Matoba, T. J. Naughton, Y. Frauel, N. Bertaux, and B. Javidi, "Real-time three-dimensional object reconstruction by use of a phase-encoded digital hologram," Appl. Opt. 41, 6187-6192 (2002).

22. T. J. Naughton and B. Javidi, "Compression of encrypted three-dimensional objects using digital holography," Opt. Eng. (Bellingham) 43, 2233-2238 (2004).

23. B. Javidi, P. Ferraro, S.-H. Hong, S. De Nicola, A. Finizio, D. Alfieri, and G. Pierattini, "Three-dimensional image fusion by use of multiwavelength digital holography," Opt. Lett. 30, 144-146 (2005).

24. I. Yamaguchi, K. Yamamoto, G. A. Mills, and M. Yokota, "Image reconstruction only by phase in phase-shifting digital holography," Appl. Opt. 45, 975-983 (2006).

25. A. E. Shortt, T. J. Naughton, and B. Javidi, "A companding approach for nonuniform quantization of digital holograms 
of three-dimensional objects," Opt. Express 14, 5129-5134 (2006).

26. E. Darakis and J. J. Soraghan, "Compression of interference patterns with application to phase-shifting digital holography," Appl. Opt. 45, 2437-2443 (2006).

27. E. Darakis and J. J. Soraghan, "Use of Fresnelets for phase-shifting digital hologram compression," IEEE Trans. Image Process. 15, 3804-3811 (2006).

28. F. Charrière, N. Pavillon, T. Colomb, C. Depeursinge, T. J. Hegera, E., A. D. Mitchell, P. Marquet, and B. Rappaz, "Living specimen tomography by digital holographic microscopy: morphometry of testate amoeba," Opt. Express 14, 7005-7013 (2006).
29. Y. Frauel, E. Tajahuerce, M.-A. Castro, and B. Javidi, "Distortion-tolerant three-dimensional object recognition with digital holography," Appl. Opt. 40, 3887-3893 (2001).

30. I. T. Nomura, A. Okazaki, M. Kameda, Y. Morimoto, and B. Javidi, "Image reconstruction from compressed encrypted digital hologram," Opt. Eng. (Bellingham) 44, 075801 (2005).

31. T. J. Naughton, J. B. McDonald, and B. Javidi, "Efficient compression of Fresnel fields for Internet transmission of three-dimensional images," Appl. Opt. 42, 4758-4764 (2003). 